\title{
EVALUACIÓN DEL ACEITE ESENCIAL Y ESTUDIO DE CONSERVACIÓN EN FRIO DEL Cymbopogon Citratus CULTIVADO EN LA REGIÓN DEL QUINDÍO
}

\section{EVALUATION OF THE ESSENTIAL OIL AND STUDY OF COLD CONSERVATION OF Cymbopogon citratus CULTIVATED IN THE REGION OF THE QUINDÍO}

\author{
Eunice Ríos Vásquez
}

Grupo de Investigación Agroindustria de Frutas Tropicales, Programa de Química, Universidad del Quindío.

Fecha de recibido: Febrero 3 de 2010

Fecha de aceptado: Junio 9 de 2010

Correspondencia: Programa de Química, Universidad del Quindío, Av. Bolivar calle 12 norte, Armenia Quindío. Correo electrónico: erios@uniquindio.edu.co.

\section{RESUMEN}

El estudio del Cymbopogon citratus conocido también como "limoncillo", fue dividido en dos partes: inicialmente se realizó la extracción del aceite esencial de sus hojas frescas, secadas por aire caliente (SAC) y secadas por invernadero (SI), utilizando dos métodos de extracción como la de destilación por arrastre con vapor (DAV) y la hidrodestilación asistida por microondas (MWHD), obteniendo los mejores rendimientos por el método de MWHD con las hojas secadas por SAC y en un tiempo de 90min., la evaluación del aceite por cromatografía de gases-espectrometría de masas (GC-MS) mostró que los compuestos mayoritarios en el aceite son el geranial y el neral; la segunda parte fue el estudio de la conservación de las hojas del C. citratus en un ambiente frio $\left(12^{\circ} \mathrm{C}\right)$ y empacados en bolsas de aluminio y frascos de vidrio, monitoreando los parámetros de acidez, $\mathrm{pH}$, actividad de agua y color, encontrándose que no hay diferencias significativas entre los envases de vidrio y aluminio, por lo cual se recomienda usar las bolsas de aluminio para su almacenamiento y conservación, debido a sus facilidades en cuanto a volumen yeconomía.

Palabras Claves: Aceite Esencial, Cymbopogon citratus, Conservación en Frio.

\section{ABSTRACT}

The study of Cymbopogon citratus also known as "lemon grass", was divided into two parts: the extraction of essential oil from fresh leaves, dried by hot air (DHA) and dried in a greenhouse (DG) using two extraction methods such as steam distillation (SD) and microwave-assisted hydrodistillation (MWHD), obtaining the best performances by the method of MWHD with the leaves dried by DHA in a time of 90min. Evaluation of oil by gas chromatography-mass spectrometry (GC-MS) showed that the main compounds in the oil are geranial and eral. The second part was to study the conservation of the leaves of $C$. citratus in a cold environment $\left(12^{\circ} \mathrm{C}\right)$ and packaged in foil pouches and glass flasks, monitoring the parameters of acidity, pH, water activity and color, finding no significant difference between glass and aluminum containers, so it is recommended to use foil pouches for storage and maintenance, due to its facilities in terms of volume and economy.

Keywords: Cold Conservation, Cymbopogon citratus, Esential Oil.

\section{INTRODUCCIÓN}

Los productos naturales, como es el caso de las plantas aromáticas y medicinales, han sido ampliamente utilizados desde tiempos atrás, bien sea como alimento, medicamento, agente conservante, etc. (1). Las plantas medicinales constituyen un grupo de gran interés debido a los principios activos presentes en ellas y que les confieren propiedades medicinales, conservantes, bactericidas y culinarias. Actualmente la medicina tradicional esta siendo en muchos aspectos complementada por la medicina natural existiendo una gran demanda de este tipo de plantas, de los aceites esenciales y otros productos procedentes de las mismas, constituyendo su cultivo como una alternativa adicional para el productor agrícola o foresta (2).
Los aceites esenciales son mezclas de sustancias orgánicas volátiles, pertenecientes a diferentes clases de compuestos, e.g. hidrocarburos, ésteres, alcoholes, aldehídos, algunos ácidos, fenoles y sus derivados, lactonas, etc.; todos, son productos de largas cadenas de biosíntesis vegetal, los llamados metabolitos secundarios de las plantas. Todos los órganos pueden contener esencia (flores, cortezas, raíces, frutos, leños, hojas, semillas). La composición de una esencia puede cambiar en la época de la recolección, el lugar geográfico o pequeños cambios genéticos. Son sustancias químicas que ejercen las funciones de auto-defensa y de atracción de polinizadores. Su uso farmacéutico se fundamenta en sus propiedades fisiológicas: el perfume y el sabor; las propiedades desinfectantes y la acción bactericida y antibiótica (3). 
Los aceites esenciales de plantas aromáticas y medicinales presentan bioactividades notables como propiedades antifúngicas, antibacteriales y antioxidantes, atrayendo la atención de químicos de productos naturales y de importantes sectores de la industria farmacéutica, de perfumes, cosmética y de alimentos, entre otras, por sus posibles y viables aplicaciones (4).

El Cymbopogon citratus, conocido también popularmente como limoncillo, es una de las 120 especies perteneciente a la familia de las Gramineae. Se cultiva en regiones tropicales y subtropicales como planta medicinal y como especia, esta contiene un $3 \%$ en aceite esencial (5), y es usado en perfumería, cosméticos, en la industria farmacéutica y alimentaria. En el aceite esencial de $C$. citratus predomina el aldehído citral (mezcla de geranial y neral) alcanzando concentraciones de 75\%; este es un buen analgésico, antipirético e insecticida (6).

De sus partes aéreas se han aislado flavonoides, ácido caféico, ácido p-cumárico, fructosa y sacarosa, y está reportado en el Vademécum Colombiano de Plantas Medicinales (7).

\section{MATERIALES Y MÉTODOS}

\section{Materia prima}

El material vegetal para el análisis fue aportado por la asociación bioagroindustrial (ASOBAI), cultivados en el corregimiento de Pueblo Tapao, Municipio de Montenegro en el Departamento del Quindío.

La humedad de las hojas fue tomada según el Método A.O.A.C. 930.15/90, pesando una cantidad constante de muestra en una cápsula de porcelana previamente tarada, se llevó a un horno de aire caliente a $110^{\circ} \mathrm{C}$ por $2 \mathrm{~h}$, transcurrido este tiempo se pesaron las cápsulas y se llevaron al desecador por una hora, se pesaron e introdujeron al horno por $1 / 2$ hora, se pesaron de nuevo las cápsulas y se repitió el procedimiento anterior hasta alcanzar peso constante.

El contenido de humedad (Hb.h.\%) se calculó de acuerdo con la siguiente ecuación:

$$
H_{\text {B.H. }}(\%)=\frac{W_{\text {Humedo }}-W_{\text {Seco }}}{W_{\text {Humedo }}} \times 100
$$

\section{Extracción y Caracterización del Aceite Esencial}

El aceite esencial fue extraído a partir de las hojas del material vegetal en estado fresco y deshidratado (secado por aire caliente a $35^{\circ} \mathrm{C}$ (SAC) y secado por invernadero (SI) por los métodos de hidrodestilación simple (HD) y asistida por microondas (MWHD), determinando para cada método el tiempo ideal para su extracción según sus rendimientos obtenidos.
Los aceites obtenidos fueron caracterizados en un cromatógrafo de gases con detector de espectrometría de masas (Ref. HRGC-KONIK 4000B-Q12) en modo de inyección Split $\left(1: 100\right.$ a $\left.290^{\circ} \mathrm{C}\right)$ usando helio como gas de arrastre a un flujo de $1 \mathrm{~mL} / \mathrm{min}$ y una columna RFX-SSil MS $(30 \mathrm{~m} \times 0.25 \mathrm{mmDI} 0.25 \mu \mathrm{m}$ Film) con el siguiente gradiente de temperatura: $2 \mathrm{~min}$ a $150^{\circ} \mathrm{C}$, aumento $10^{\circ} \mathrm{C} / \mathrm{min}$ hasta $320^{\circ} \mathrm{C}$ donde se mantiene $5 \mathrm{~min}$, el detector en modo de impacto electrónico (EI) inició luego de $3 \mathrm{~min}$ de inyección escaneando cada $0.5 \mathrm{seg}$ masas entre los 70 y $500 \mathrm{~m} / \mathrm{z}$. Para obtener así la separación e identificación de sus compuestos, Además de medir sus propiedades fisicoquímicas de peso específico, índice de refracción y pH según los métodos específicos de la A.O.A.C.

\section{Estudios de Conservación}

Las plantas empacadas en vidrio y bolsas de aluminio se almacenaron durante 7 días en una cámara de conservación con control de temperatura $\left(12^{\circ} \mathrm{C}\right)$ y humedad relativa $(20 \%)$, evaluando su comportamiento durante su periodo de almacenamiento teniendo en cuenta su:

- Actividad de agua (aw): se determinó con un higrómetro de punto de rocío (Aqualab Decagon modelo 3TE) de cuatro cifras significativas, realizando por triplicado el análisis de cada muestra.

- $\mathrm{pH}$ : se midió en un pHmetro (IQ scientific modelo IQ 240), con un electrodo para sólidos previamente calibrado con soluciones tampón de $\mathrm{pH} 2,4$ y 7.

- Acidez Titulable (Método A.O.A.C. 942.15/90 Modificado): Se pesó una cantidad constante de muestra homogenizada y se diluyó a $100 \mathrm{~mL}$ con agua destilada previamente hervida. Esta solución fue titulada con $\mathrm{NaOH} 0,1 \mathrm{~N}$ estándar usando 5 gotas de solución alcohólica de fenolftaleina $1 \%$ como indicador.

El valor de la acidez titulable se expresó en gramos de ácido cítrico por $100 \mathrm{~g}$ de muestra según la ecuación:

$$
\text { Acidez }(\text { mgAcide Citrico } / 100 \text { g Muestra })=\frac{\left(V_{\text {NadoH }}\right)(700)}{g_{\text {Muestra }}}
$$

-Color: Las variaciones en el color se determinaron utilizando un colorímetro (Minolta modelo $\mathrm{CM}-41$ ) con observador a $10^{\circ}$ e iluminante D65, mediante el sistema de medición objetivo $\mathrm{CIE}-\mathrm{L}^{*} \mathrm{a} * \mathrm{~b} *$, evaluando la luminosidad $\left(L^{*}\right),\left(a^{*}\right)$ y $\left(b^{*}\right)$, saturación $(C)$ y matiz $\left(h^{\circ}\right)$.

\section{RESULTADOS Y DISCUSIÓN}

\section{Obtención del Aceite Esencial}

Los rendimientos de extracción utilizando DAV y MWHD, se muestran en la figura 1.

Rev. Invest. Univ. Quindío (20): 24-28. Armenia - Colombia 
a) por DAV

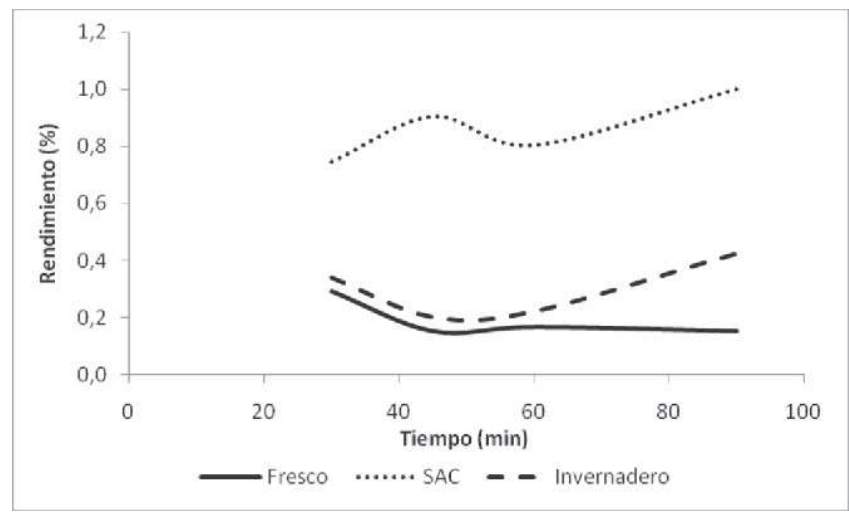

Figura 1. Rendimientos de Extracción de Aceite Esencial
b)Por MWHD

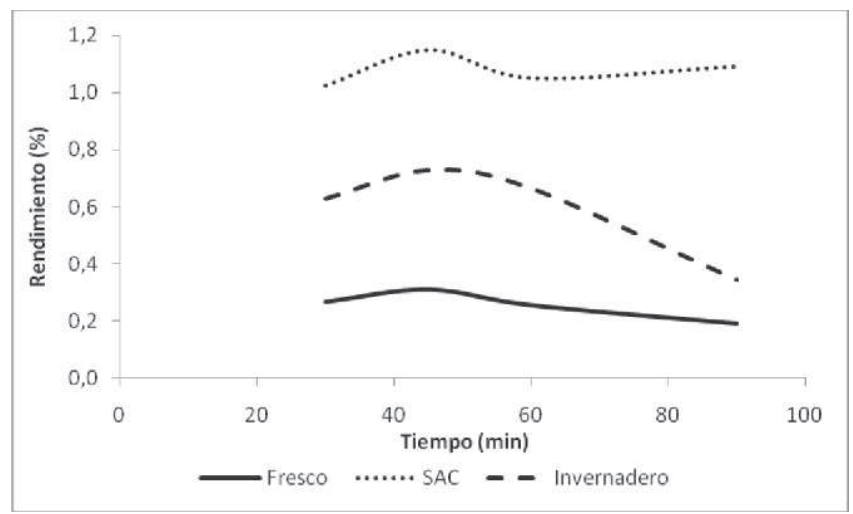

MWHD, mostrando principalmente compuestos terpénicos oxigenados.

a) Por DAV

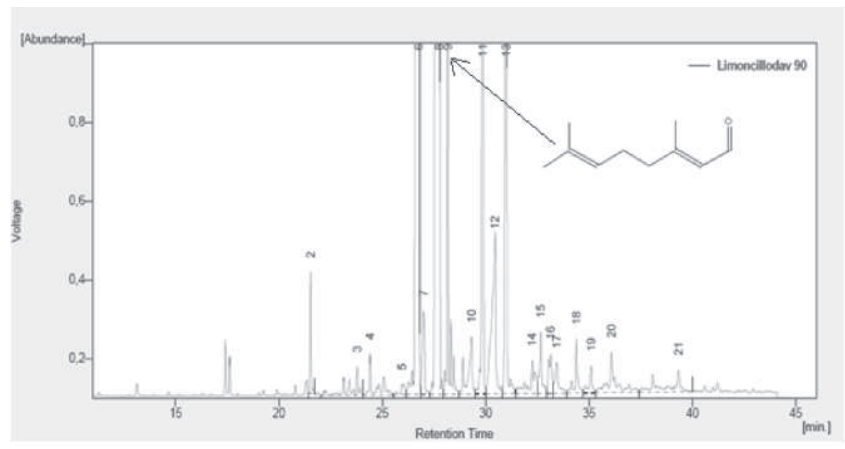

b) Por MWHD

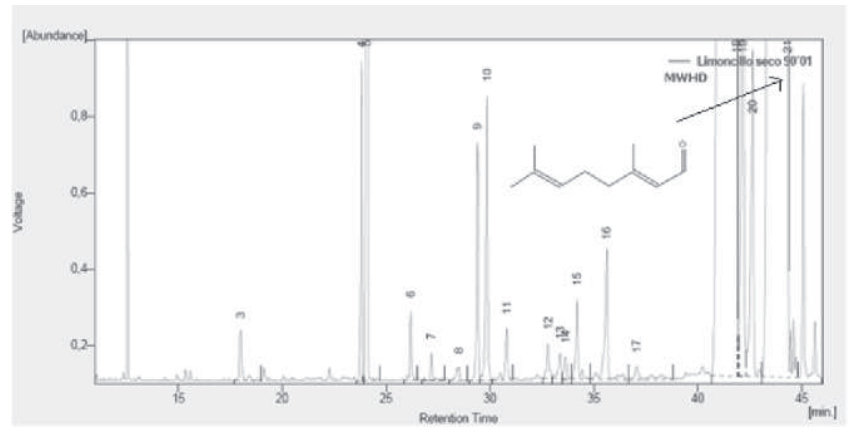

Figura 2. Cromatogramas del Aceite Esencial extraido del Cymbopogon citratus
Los compuestos encontrados en mayor proporción en el aceite esencial fueron el geranial (20,1\% por DAV y $25,5 \%$ por MWHD) y el neral (18,1\% por DAV y $21,5 \%$ por MWHD), se puede observar en la tabla 2 que en la extracción MWHD se presenta mayores abundancias relativas para la mayoría de los compuestos estudiados. 
Tabla 2. Compuestos mayoritarios del aceite esencial obtenido por MWHD y DAV en un tiempo de extracción de 90 minutos

\begin{tabular}{c|c|c|c}
\hline $\begin{array}{c}\text { Método de } \\
\text { extracción }\end{array}$ & $\begin{array}{c}\text { Compuestos mayoritarios, } \\
\text { pico }\end{array}$ & $\begin{array}{c}\text { Área - \% } \\
\text { abundancia }\end{array}$ & Índice de retención (min) \\
\hline MWHD & Geranial (Citral A), pico 21 & 25.5 & 23.875 \\
& Neral(Citral B), pico 18 & 21.5 & 24.799 \\
DAV & Geranial (Citral A), pico 8 & 20.1 & 24.556 \\
& Neral(Citral B), pico 6 & 18.1 & 23.901 \\
\hline
\end{tabular}

\section{Estudios de Conservación en Frio}

Luego de 7 días de análisis de las muestras empacadas en frascos de vidrio y bolsas de aluminio, se puede notar que la aw (Fig. 3) aumenta para cada empaque con respecto al tiempo posiblemente debido a los procesos de transpiración normales de la planta que aumentan la cantidad de agua libre en la misma.

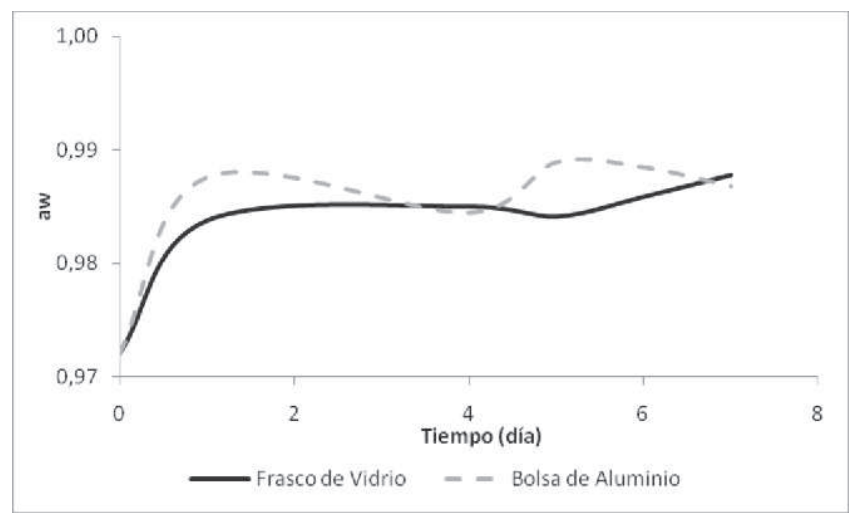

Figura 3. Actividad de Agua durante la Conservación

La perdida de ácidos orgánicos durante la conservación (Fig. 4) debido posiblemente a los exudados de la transpiración de la planta generan un aumento en el $\mathrm{pH}$ de las plantas con respecto al tiempo (Fig. 5).

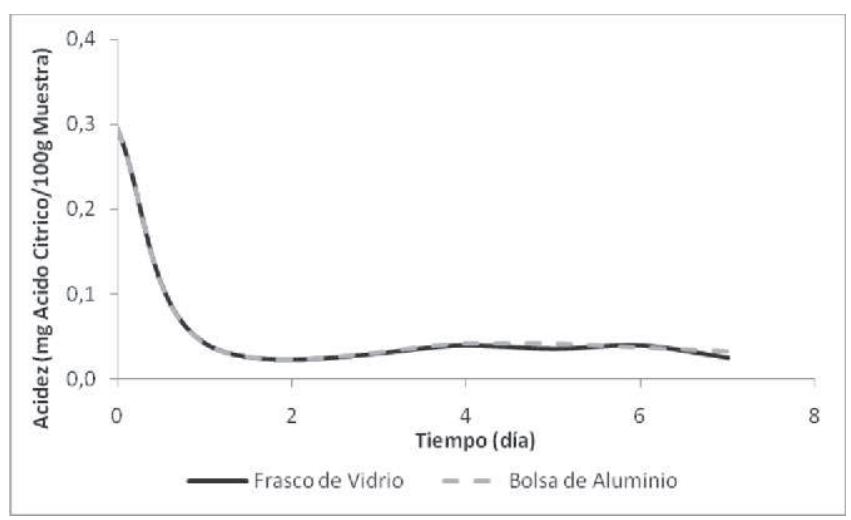

Figura 4. Acidez durante la Conservación.

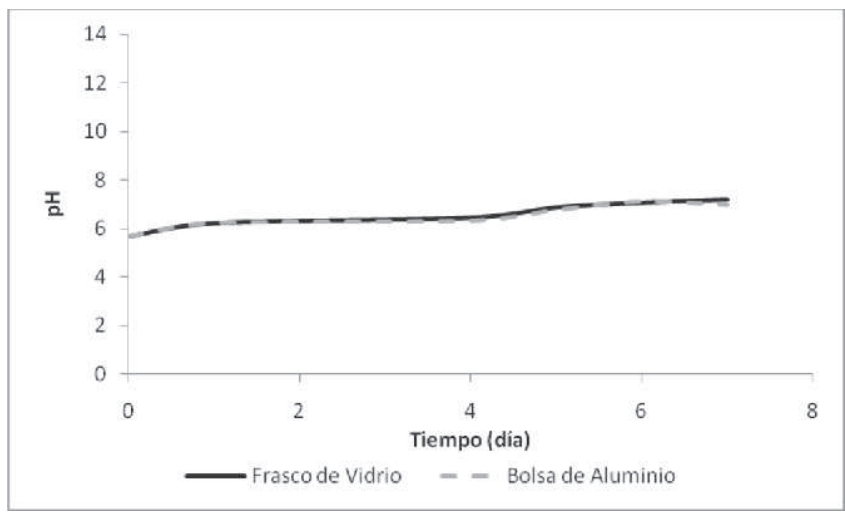

Figura 5. pH durante la Conservación.

Los cambios en las variables colorimetricas se muestran en la figura 6. La luminosidad (Fig. 6a) de las hojas no se ve afectada con el paso del tiempo ni tampoco se presentan diferencias significativas para los empaques de vidrio y aluminio debido probablemente a que la temperatura de refrigeración retarda los procesos de oxidación propios de la planta que favorecen el oscurecimiento de la misma. Las variables $a^{*}$ y $b^{*}$ (Fig. 6b) reflejan que con el paso del tiempo las muestras tienden a cambiar su color hacia el amarillo ( $b^{*}$ positivo), lo que tambien se podia evidenciar fisicamente; el incremento en el croma (Fig. 6c) y el descenso del matiz (Fig. 6d) indican en concordancia con a* y b* que se presentó un cambio en el color hacia un tono amarillo verdoso con una mayor saturación de color.

a)

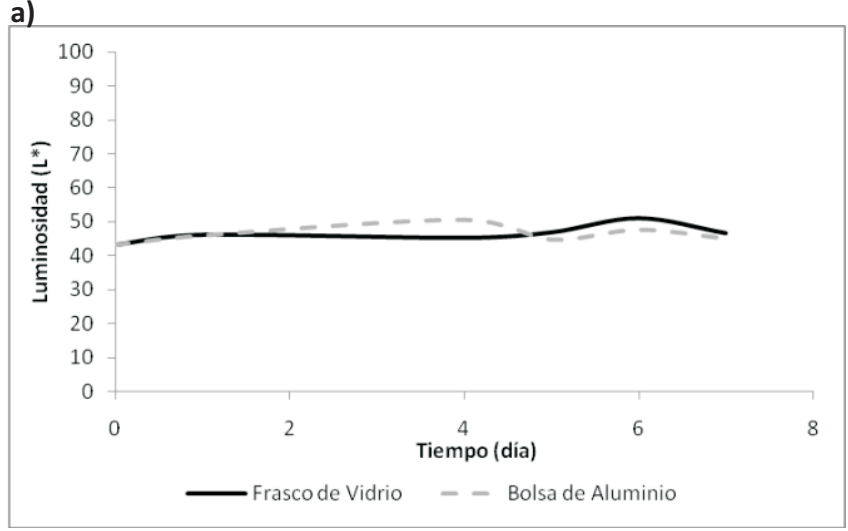


b)

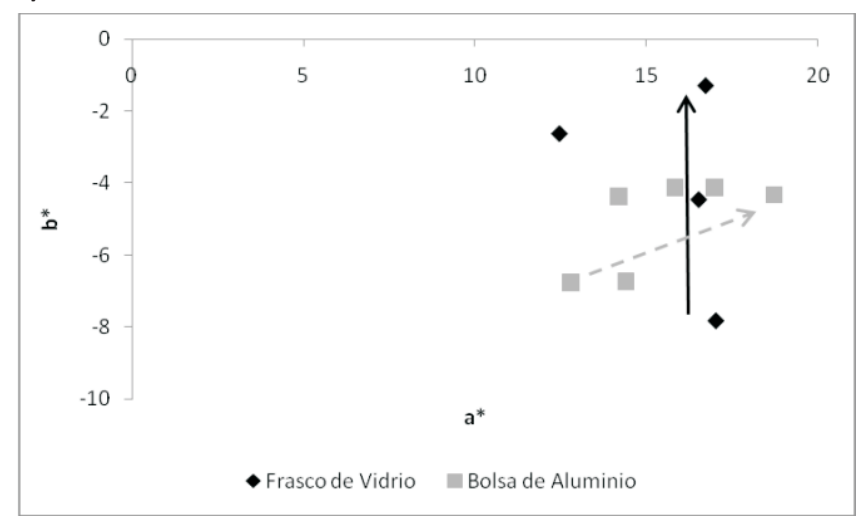

d)

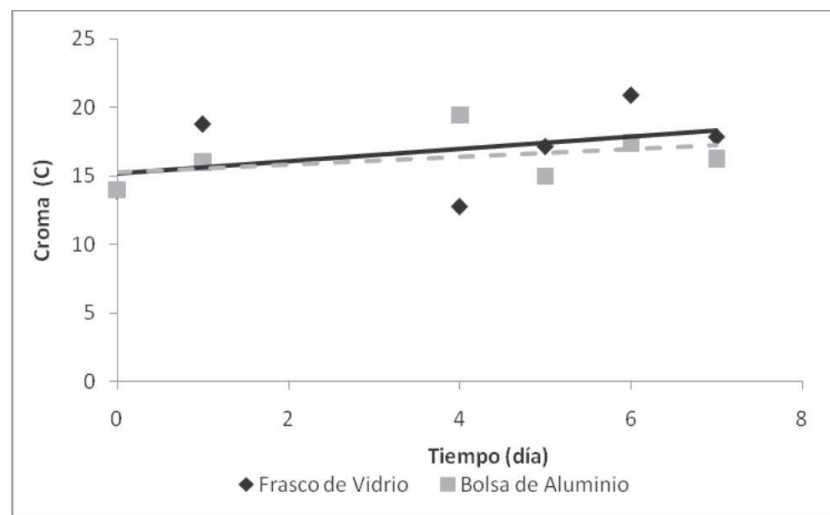

Figura 6. Variables Colorimetricas durante la Conservación: a) $L^{*}$, b) $a^{*}$ y b*, c) $C^{*}$, d) $h^{\circ}$. c)

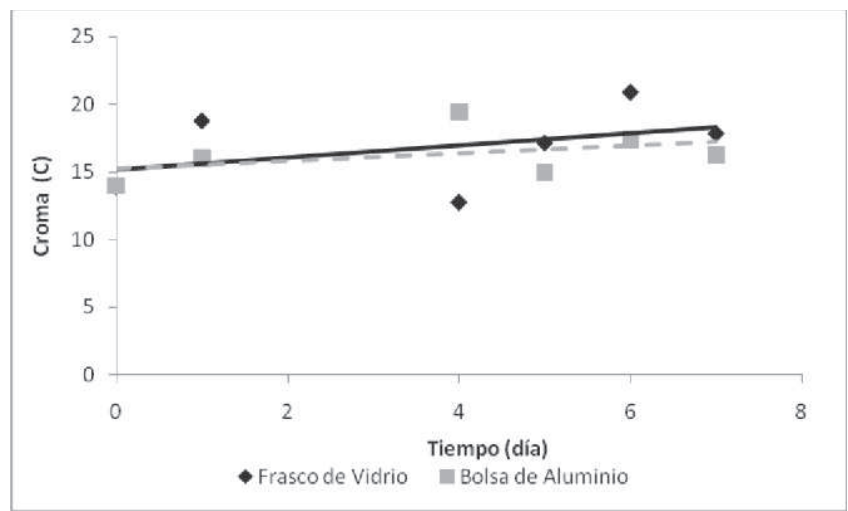

\section{CONCLUSIONES}

La extracción del aceite esencial es más eficiente cuando las hojas de cymbopogon citratus son secadas por aire caliente (SAC) antes del proceso, obteniendo $1.29 \%$ utilizando la técnica de MWHD durante $90 \mathrm{~min}$. Esta Técnica es ideal para obtener los mejores rendimientos de extracción sin degradaciones de compuestos aromáticos. El quimiotipo presente es citral, el cual se encuentra conformado por sus dos isómeros geométricos, el geranial y el neral.

La conservación en frio del material vegetal por una semana muestra que no hay diferencias significativas entre el empaque en vidrio y las bolsas de aluminio, por lo que se recomienda usar bolsas de aluminio para su conservación por su facilidad para el transporte y economía en comparación con el vidrio.

\section{AGRADECIMIENTOS}

Agradecemos a la Asociación Bioagroindustrial del Quindío (ASOBAI) por sus aportes y colaboración con el material vegetal. Además se agradece al personal del laboratorio Diseño de Nuevos Productos del programa de Química de la Universidad del Quindío por toda su cooperación.

\section{BIBLIOGRAFÍA}

1. Stachenko, E., Jaramillo B.E. y Martinez J.R. "Comparison of different extraction methods for the analysis of volatile secondary metabolites of Lippia alba (Mill.) N.E. Brown, grown in Colombia, and evaluation of its in vitro antioxidant activity", En Journal of Chromatography A, 2004. 1025, 93-103.

2. Schroeder, M.A., Lopez A.E. y Martínez G.C. (2004). "Resultados Preliminares del Analisis Foliar de Lippia alba (Mill.) N.E. Brown, Pluchea Sagittalis (Lamb) cab., Petiveria alliace L. y Ocimun Selloi Benth". En Comunicaciones Cientificas y Tecnologicas, Universidad Nacional del Nordeste, A-035.

3. Minami, K. (1999). "Relatório do Estágio Supervisionado Produção Vegetal-II: manejo e produção de plantas medicinais e aromáticas". Universidad de São Paulo, Escuela Superior de Agricultura "Luíz de Queiroz", Departamento de Producción Vegetal.

4. Acevedo, A.M., Castañeda M.L., Blanco K., Cárdenas C.Y., Reyes J.A., Kouznetsov V. y Stashenko, E. “Composición y Actividad Antioxidante de Especies Aromáticas y Medicinales con Alto Contenido de Timol y Carvacrol". En Scientia et Technica, 2007. 8(33), 125-128.

5. Quintero, A., Gonzales N. y Vera A. “Obtención y análisis cromatográfico del aceite esencial de Cymbopogon citratus (Limonaria mem)". En Inst. Biol. Exper,1999. 211-214.

6. Fomegrag, R. y Jimenez S. (2007). “Plantas medicinales aprobadas de Colombia, Segunda Edición”. Editorial Universidad de Antioquia, Medellín Colombia.

7. Vademécum Colombiano de Plantas Medicinales, (2008). Ministerio de Protección Social, 130-131.

8. Ríos, E., Giraldo G.A., León D.F. y Moreno A. “Estudio del perfil de compuestos volátiles de los rizomas de Curcuma longa L. Cultivada en el departamento del Quindío - Colombia”. En Revista de Investigaciones Universidad del Quindío; 2008. (18), 32-37.

9. AOAC. (1997). "Official methods of analysis, 16th Edición, 3a Revisión". Association of official Analytical Chemists, Washington D.C., USA.

Rev. Invest. Univ. Quindío (20): 24-28. Armenia - Colombia 